\title{
Avaliação de métodos automáticos de limiarização para imagens de plantas de milho atacadas por Spodoptera frugiperda ${ }^{1}$
}

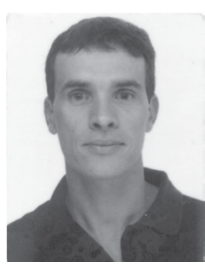

\author{
Darly G. de Sena Júnior², Francisco de A. de C. Pinto ${ }^{3}$, Reinaldo L. Gomide ${ }^{4}$ \& Mauri M. Teixeira ${ }^{5}$ \\ 1 Parte da Dissertação de Mestrado apresentada pelo primeiro autor ao Programa de Pós-Graduação em Engenharia \\ Agrícola da Universidade Federal de Viçosa; \\ 2 DEA/UFV. CEP 36571-000, Viçosa, MG. Fone: (31) 3899-1881. E-mail: sena@vicosa.ufv.br (Foto) \\ 3 DEA/UFV. E-mail: facpinto@ufv.br \\ 4 EMBRAPA Milho e Sorgo. E-mail: gomide@cnpms.embrapa.br \\ ${ }^{5}$ DEA/UFV. E-mail: mauri@ufv.br
}

Protocolo 29 - 22/3/2002 - Aprovado em 25/7/2003

\begin{abstract}
Resumo: Um dos passos fundamentais no processamento de imagens para um sistema de visão artificial é a segmentação dos objetos de interesse na cena, e um dos métodos mais utilizados é a limiarização, em especial quando o objetivo é agrupar os pixels em duas classes. Neste método, o valor do limiar determina o número de pixels atribuídos a cada classe, além de influenciar a dimensão e a forma dos objetos nas imagens segmentadas. A utilização de métodos automáticos para definição do limiar, não só evitaria a influência de operadores mas, também, tornaria mais rápida a escolha dos limiares no campo, onde a variação da iluminação influencia os valores dos pixels. Este trabalho objetivou implementar e avaliar dois métodos automáticos de limiarização para identificação de plantas de milho atacadas pela lagarta do cartucho. Foram utilizadas imagens de plantas atacadas e não-atacadas, em três épocas, correspondendo a diferentes dias após a infestação. As plantas foram reunidas em três grupos de 10, sendo as imagens de cada grupo obtidas sob uma intensidade luminosa diferente. As imagens processadas com o índice do excesso de verde normalizado foram limiarizadas, automaticamente, e comparadas com a limiarização manual das mesmas imagens. Os resultados obtidos pelos dois métodos automáticos de limiarização foram satisfatórios, apresentando média acima de $99 \%$ de exatidão global, evidenciando-se, portanto, que ambos os métodos têm potencial para serem utilizados em um sistema de identificação de plantas de milho atacadas pela lagarta do cartucho.
\end{abstract}

Palavras-chave: processamento de imagens, visão artificial, agricultura de precisão

\section{Evaluation of automatic thresholding methods for images of Spodoptera frugiperda affected corn plants}

\begin{abstract}
A fundamental step in image processing for machine vision is the scene segmentation and one of the most used methods is the threshold, especially when one desires to cluster the pixels in two classes. The image threshold affects the number of pixels in each class and the object shape and dimension. An automatic thresholding method not only could avoid operator's influence but also could speed up the threshold value definition in the field where light variation influences the pixels values. In this work, two automatic thresholding methods were evaluated for use in a machine vision system that identifies damaged corn plants by the armyworms. The images used were of damaged and not damaged corn plants in three stages. Three groups of 10 plants were collected to take the images of each group under different light intensities. The images processed with the excess green index were thresholded with the automatic methods and compared with the manual thresholding methods. The results of both automatic methods were good, with a mean accuracy higher than $99 \%$, showing the potential of the system for identifying the damaged corn plants.
\end{abstract}

Key words: image processing, machine vision, precision farming

\section{INTRODUÇÃO}

A necessidade de aumento da produção agrícola, em virtude do crescimento da população, promoveu o desenvolvimento da mecanização, o que permitiu o cultivo de grandes áreas. As parcelas foram incorporadas em talhões, devido à impossibilidade de manejá-las de acordo com suas características particulares com manejo e aplicação de insumos definidos pela média. A partir da disponibilidade de técnicas de localização precisas com custo acessível, aliadas ao desenvolvimento de 
sensores e sistemas eletrônicos aplicáveis a máquinas agrícolas, descortinou-se a possibilidade de manejo em sítio específico dessas áreas. Este manejo, de acordo com as exigências locais, é o que vem sendo denominado agricultura de precisão (AP) pelos técnicos.

No Brasil, a principal praga na cultura do milho é a lagarta do cartucho (Spodoptera frugiperda) (Fancelli \& Dourado Neto 2000). Perdas de até $42 \%$ da produção em parcelas de milho atacadas por esse inseto, em relação a parcelas não atacadas, já foram detectadas (Oliveira, 1987). A lagarta do cartucho vem sendo controlada com aplicação de inseticidas na área total da lavoura, quando a população atinge o nível de ação, ou seja, o dano provocado pela praga justifica, economicamente, a utilização de medidas de controle. Para controle, utilizam-se principalmente métodos convencionais, por meio de pulverizador costal ou tratorizado (Carvalho, 1987), tratamento de sementes (Oliveira \& Silva, 1994) e via água de irrigação (Viana \& Costa, 1994). O nível de infestação é estimado contando-se as plantas com injúrias provocadas pela lagarta, em amostragem realizada ao acaso, na lavoura, por pessoal treinado. Embora poucos trabalhos tenham sido publicados visando ao controle localizado de pragas em culturas, a lagarta do cartucho apresenta um grande potencial para este tipo de controle, devido à sua baixa mobilidade na cultura.

Um dos métodos estudados para viabilizar a coleta de dados para AP é a utilização de sistemas de visão artificial (SVA). As principais características desses sistemas são a reprodutibilidade, a possibilidade de se operar em praticamente todo o espectro de radiações eletromagnéticas e a capacidade de medir objetivamente a cor de objetos (Marques Filho \& Vieira Neto, 1999). Esses sistemas se constituem, basicamente, de uma câmera conectada a um computador por meio de uma placa de aquisição de vídeo e de programas computacionais específicos de processamento de imagens, para extração das informações desejadas. A partir dessas informações, o sistema pode gerar um sinal para controle de máquinas, ou armazenar os dados para utilização posterior como, por exemplo, a elaboração de um mapa de prescrição (Pinto et al., 2002).

Em um SVA, é muito importante a subdivisão da imagem em suas partes constituintes ou objetos, técnica conhecida como segmentação. Para isto, é utilizada a limiarização, especialmente quando os valores dos pixels apresentam uma distribuição bimodal e o objetivo é agrupá-los em duas classes, criando-se uma imagem binária, caso em que um valor é estabelecido (limiar) e os pixels que apresentam valores inferiores e superiores ao limiar são agrupados em duas classes diferentes. A seguir, são extraídas das imagens binárias, as características que serão utilizadas para classificar os objetos presentes. Medições adequadas de objetos, em imagens, requerem o uso de um método de limiarização confiável e acurado, devido à influência no número de pixels atribuídos a cada classe, a dimensão e a forma dos objetos segmentados. Freqüentemente, a seleção do método é feita com base em experimentação e avaliação (Yang et al., 1995). O ideal seria a utilização de um método automático, para evitar influência de operadores. Contudo, em muitos casos as imagens limiarizadas manualmente são utilizadas como segmentação de referência para comparação com o resultado do algoritmo (Yang et al., 2001).
A intensidade luminosa é um fator que influencia diretamente os valores numéricos dos pixels em uma imagem digital, uma vez que esses valores representam a energia refletida pelos objetos e incidente no sensor da câmera. Este é um dos desafios enfrentados na utilização de SVA para agricultura de precisão, em razão da variação dos níveis de iluminação e das sombras presentes na imagem (Steward \& Tian, 1998). Outro fator que dificulta a segmentação da imagem é a variabilidade de cor da luz, de acordo com o dia e com a época do ano (Tian \& Slaughter, 1998). Batlle et al. (2000) em revisão sobre estratégias para reconhecimento de objetos em imagens coloridas obtidas em ambientes externos, afirmaram que os resultados obtidos por SVA, nessas condições, precisam ser melhorados, pois os problemas só estão solucionados para cenas simples.

A segmentação pode ser facilitada com a normalização, uma técnica muito utilizada para reduzir os efeitos da variação de iluminação e a variância nos valores dos pixels na imagem. Nesta técnica, os valores originais dos pixels, em mais de uma banda da imagem, são combinados em índices para gerar uma nova imagem (Andreadis \& Tsalides, 1997). Os índices são amplamente utilizados na área de sensoriamento remoto, no intuito de reduzir a diferença de brilho em materiais idênticos, causados por diferenças no relevo, sombras ou mudanças sazonais no ângulo e intensidade da iluminação solar. Além disso, permite realçar características de interesse e reduzir o número de bandas necessárias à interpretação, concentrando a informação (Jensen, 1996). Woebbecke et al. (1995) testaram diversos índices de cor para distinção de plantas do solo e resíduos, em condições de iluminação natural, em imagens sombreadas e não-sombreadas, em que os melhores resultados foram obtidos com os índices de matriz modificado e o excesso de verde; no entanto, o primeiro exigiu mais tempo de processamento. Meyer et al. (1998) também utilizaram o índice de excesso de verde normalizado, associado a técnicas de avaliação de textura, para identificar quatro espécies de plantas daninhas, mostrando-se eficiente para classificar o solo e plantas daninhas, com acurácia superior a 99\%.

O objetivo deste trabalho foi implementar e avaliar dois métodos automáticos de limiarização, iterativo (Yang et al., 2001) e bayesiano (Gonzales \& Woods, 1992) utilizando-se imagens processadas de plantas de milho atacadas e não-atacadas pela lagarta do cartucho (Spodoptera frugiperda).

\section{MATERIAL E MÉTODOS}

Imagens de 15 plantas atacadas e 15 plantas não-atacadas pela lagarta do cartucho foram obtidas em três épocas distintas e sob três intensidades de iluminação, em condições de casade-vegetação, nas dependências da EMBRAPA Milho e Sorgo, em Sete Lagoas, MG. As plantas foram reunidas em grupos de 10 (5 atacadas e 5 não-atacadas) assim como as imagens de cada grupo obtido sob uma intensidade de iluminação, perfazendo o total de 30 imagens para cada estádio.

O trabalho foi conduzido utilizando-se 30 vasos de $15 \mathrm{~L}$ com solo peneirado e adubado com a formulação NPK 4-14-8, na quantidade de $3,00 \mathrm{~kg} \mathrm{t}^{-1}$ de solo, mantidos em casa-devegetação. Em cada vaso foram semeadas três sementes do híbrido triplo HT971011, no dia 26/6/2001. As plantas emergiram 
no dia 3/7/2001 e, após uma semana, foram desbastadas para uma planta por vaso. Quinze dias após a emergência, foram escolhidas, aleatoriamente, quinze plantas para infestação com 10 lagartas de Spodoptera frugiperda, eclodidas no dia anterior.

As plantas foram sorteadas para obtenção das imagens sob três intensidades de iluminação, aos 17, 20 e 23 dias após a emergência , correspondendo a 2, 5 e 8 dias após a infestação pela lagarta do cartucho, com a câmera posicionada $1 \mathrm{~m}$ acima das folhas mais altas das plantas. Utilizou-se uma câmera digital DUNCANTECH, modelo MS3100, conectada a um microcomputador por meio de uma placa de aquisição de vídeo da National Instruments, modelo PCI 1424. As imagens coloridas RGB foram gravadas com dimensões de 1392 (H) e 1039 (V) pixels no formato TIFF (Tagged Image File Format). A câmera utilizada trabalha com 3 CCDs ("charge-coupled device"), os quais permitem o controle do ganho e do tempo de exposição em cada sensor. Os CCDs foram destinados à coleta de imagens nas das bandas do vermelho, do infravermelho próximo e do verde e azul. A lente permitia o controle manual da abertura da íris, em que o "f-stop" foi definido em 3,5. A Tabela 1 apresenta o ganho e o tempo de exposição utilizados para cada CCD na aquisição das imagens das plantas.

Tabela 1. Ganho e o tempo de exposição para cada um dos três CCDs (Charge-coupled Device) nas bandas do vermelho (Vm), do infravermelho próximo (IV próx) e do verde e azul ( $\mathrm{Vd} / \mathrm{Az}$ ) para um "f-stop" de 3,5

\begin{tabular}{lccc}
\hline CCD & Vm & IV próx & Vd/Az \\
\hline Ganho $(\mathrm{dB})$ & 15,0 & 12,9 & 10,2 \\
Exposição $(\mathrm{ms})$ & 123,000 & 122,625 & 122,625 \\
\hline
\end{tabular}

Para obtenção das imagens, sob condições de iluminação controlada, construiu-se uma estrutura metálica com dimensões $1,50 \times 1,50 \times 2,00 \mathrm{~m}$ de altura, revestida com lona plástica, tendo uma face branca voltada para o interior da estrutura. O sistema de iluminação foi composto de seis lâmpadas incandescentes dicróicas de $50 \mathrm{~W}$ e mais quatro refletores, com lâmpadas incandescentes halógenas de $150 \mathrm{~W}$. O sistema de iluminação foi disposto de modo a fornecer uma iluminação o mais difusa possível. Para garantir que os níveis de iluminação fossem os mesmos todos os dias, as imagens foram obtidas à noite. Para obtenção das três intensidades de iluminação, os quatro refletores foram mantidos acesos e as demais lâmpadas foram acesas sucessivamente, duas a duas.

As imagens foram processadas no Laboratório de Projeto de Máquinas e Visão Artificial do Departamento de Engenharia Agrícola da Universidade Federal de Viçosa. Foi utilizado o sistema computacional MATLAB com o pacote de ferramentas para processamento de imagens. As imagens originais foram processadas com o índice do excesso de verde normalizado, de acordo com a Eq. (1):

$$
\mathrm{Evd}=\frac{2 \mathrm{Vd}-\mathrm{Vm}-\mathrm{Az}}{\mathrm{Vm}+\mathrm{Vd}+\mathrm{Az}}
$$

em que:

Evd - excesso de verde normalizado

$$
\begin{aligned}
& \mathrm{Vd} \text { - valor do pixel na banda verde } \\
& \mathrm{Vm} \text { - valor do pixel na banda vermelha } \\
& \mathrm{Az} \text { - valor do pixel na banda azul }
\end{aligned}
$$

Tendo em vista que as imagens processadas apresentavam valores entre -1 e 2, procedeu-se a uma transformação linear nos valores dos pixels, criando-se imagens em 256 tons de cinza, de acordo com a Eq. (2):

$$
\operatorname{Evd}_{\mathrm{t}}=\frac{\mathrm{Evd}_{\mathrm{o}}-\mathrm{Evd}_{\text {min }}}{\mathrm{Evd}_{\text {max }}-\mathrm{Evd}_{\text {min }}} 255
$$

As imagens coloridas originais apresentavam, em suas bordas, pixels com valores nulos, provenientes, possivelmente, de problemas na aquisição das imagens pela placa. Para não influenciar o resultado dos testes, foram eliminadas as 10 primeiras e as 10 últimas colunas de pixels das imagens, assim como as 10 primeiras e as 10 últimas linhas, reduzindo a dimensão das imagens processadas para $1372(\mathrm{H})$ por $1019(\mathrm{~V})$ pixels.

Uma rotina foi desenvolvida no MATLAB, para que cada um dos cinco operadores pudesse obter, manualmente, por tentativa, um valor de limiar que segmentasse adequadamente as classes folha e fundo nas imagens normalizadas. Esta rotina apresentava, de inicío, a imagem colorida original e uma imagem binária, criada utilizando-se um limiar baixo. O operador poderia aumentar ou diminuir o limiar, sem ter acesso ao valor, para não influenciar a limiarização das imagens subseqüentes. A cada alteração do limiar pelo operador, o programa apresentava a nova imagem binária acompanhada da imagem original. No momento em que o operador considerava a imagem binária satisfatória, o programa gravava, em um arquivo, o nome da imagem e o valor do limiar. Ao final, para cada imagem uma média aritmética foi calculada usando-se os limiares obtidos pelos cinco operadores. Esses limiares médios assim obtidos, foram utilizados para criar as imagens binárias de referência para avaliação dos métodos automáticos de limiarização.

Foram utilizados dois métodos automáticos de limiarização: iterativo (Yang et al., 2001) e bayesiano (Gonzales \& Woods, 1992). O primeiro assume que o valor do limiar ótimo pode ser obtido calculando-se a média dos valores médios dos pixels nas duas classes de um histograma bimodal. O histograma da imagem é percorrido para encontrar o valor de limiar que promove o maior grau de separação entre as duas classes. O método bayesiano considera o histograma da imagem como uma estimativa da função densidade de probabilidade do brilho. Assumindo-se que os histogramas das imagens apresentavam distribuição gaussiana bimodal, o limiar que minimiza a probabilidade de erros de classificação (limiar ótimo) foi estimado por meio da Eq. (3) (Gonzales \& Woods, 1992):

$$
\mathrm{AT}^{2}+\mathrm{BT}+\mathrm{C}=0
$$

em que:

T - valor do limiar ótimo 
Os fatores A, B e C foram calculados por meio das Eqs. (4), (5) e (6):

$$
\begin{gathered}
\mathrm{A}=\sigma_{1}^{2}-\sigma_{2}^{2} \\
\mathrm{~B}=2\left(\mu_{1} \sigma_{2}^{2}-\mu_{2} \sigma_{1}^{2}\right) \\
\mathrm{C}=\sigma_{1}^{2} \mu_{2}^{2}-\sigma_{2}^{2} \mu_{1}^{2}+2 \sigma_{1}^{2} \sigma_{2}^{2} \ln \left(\sigma_{2} \mathrm{P}_{1} / \sigma_{1} \mathrm{P}_{2}\right)
\end{gathered}
$$

em que:

$$
\begin{array}{ll}
\sigma_{1}^{2} & \text { - variância da classe } 1 \text { da imagem } \\
\sigma_{2}^{2} & \text { - variância da classe } 2 \text { da imagem } \\
\mu_{1} & \text { - média dos níveis de cinza da classe } 1 \\
\mu_{2} & \text { - média dos níveis de cinza da classe } 2 \\
\mathrm{P}_{1} & \text { - probabilidade de ocorrência da classe } 1 \\
\mathrm{P}_{2} & \text { - probabilidade de ocorrência da classe } 2
\end{array}
$$

As médias, as variâncias e as probabilidades de ocorrência das classes na imagem, foram estimadas minimizando-se a função do erro-médio quadrático entre a função gaussiana bimodal e os valores do histograma da imagem. A minimização foi realizada utilizando-se o método do gradiente descendente (Mateus \& Luna, 1986).

Os valores dos limiares foram comparados por meio de uma regressão linear sem intercepto entre os valores do limiar manual e dos métodos automáticos de limiarização (Yang et al. 2001); além disso, as imagens binárias de referência e as imagens binárias das mesmas plantas, criadas com os dois métodos automáticos de limiarização, foram comparadas utilizando-se uma rotina desenvolvida no MATLAB, para criar a matriz de erros ou de contingência. Por meio dessa matriz, é possível verificar a exatidão da classificação em cada classe, acessandose os erros de comissão e de omissão (Jensen, 1996). Os erros de comissão das classes foram calculados por meio da Eq. (7):

$$
\mathrm{EC}_{\mathrm{i}}=\frac{\mathrm{pe}_{\mathrm{i}}}{\mathrm{pc}_{\mathrm{i}}} 100
$$

em que:

$\mathrm{EC}_{\mathrm{i}}$ - erro de comissão da classe i

$\mathrm{pe}_{\mathrm{i}}$ - número de pixels da classe i atribuídos a outra classe

$\mathrm{pc}_{\mathrm{i}}$ - número total de pixels da classe i na imagem binária de referência

Utilizando-se o número total de pixels corretamente classificados na imagem, a diagonal da matriz de erros, calculou-se a exatidão global da classificação (EG):

$$
\mathrm{EG}=\frac{\mathrm{pc}}{\mathrm{pt}} 100
$$

em que:

EG - exatidão global da classificação

pc - número de pixels corretamente classificados

pt - número total de pixels na imagem
Foram criadas, ainda, as imagens-diferença, comparandose as imagens resultantes da limiarização automática com as imagens resultantes da limiarização manual. Nessas imagens binárias, os pixels, classificados da mesma forma pelos dois métodos, são atribuídos a uma classe, enquanto os demais pixels o são a outra.

\section{RESULTADOS E DISCUSSÃO}

A Figura 1 ilustra a regressão linear entre os valores dos limiares manuais e automáticos. Os resultados dos dois métodos foram semelhantes, uma vez que as regressões apresentaram valores de $\beta$ próximos $(0,8426$ e 0,8917$)$ e $r^{2}$ maior que 0,99 .

A.

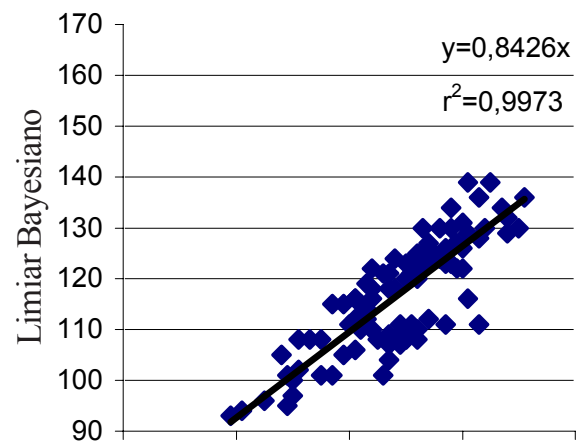

B.

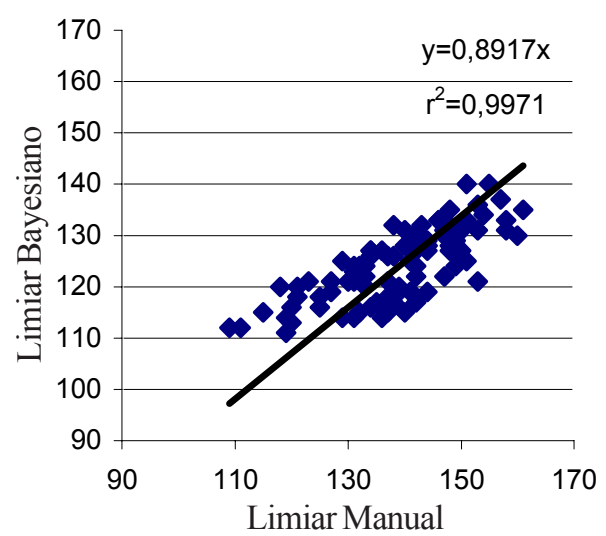

Figura 1. Regressão linear sem intercepto entre os valores do limiar manual e limiares bayesiano (A) e iterativo (B)

A Figura 2A ilustra os resultados da estatística descritiva dos valores da exatidão global das imagens binárias obtidas com o algoritmo bayesiano, considerando-se o limiar manual como referência, para plantas atacadas e não-atacadas, nas 3 épocas de obtenção das imagens, e sob as 3 intensidades de iluminação. A Figura 2B ilustra os resultados da mesma análise para os resultados do algoritmo iterativo. Os dois algoritmos apresentaram a mesma tendência de resultados, com exatidão global ligeiramente maior no caso do algoritmo iterativo, especialmente no caso mais crítico, ou seja, as plantas atacadas no estádio mais avançado ( 23 dae/8dai)*. Em todas as situações, os dois algoritmos apresentaram exatidão global acima de $97 \%$, com melhor resultado para plantas não-atacadas. Em relação às

" dae - dias após a emergência; dai - dias após a infestação 
A.
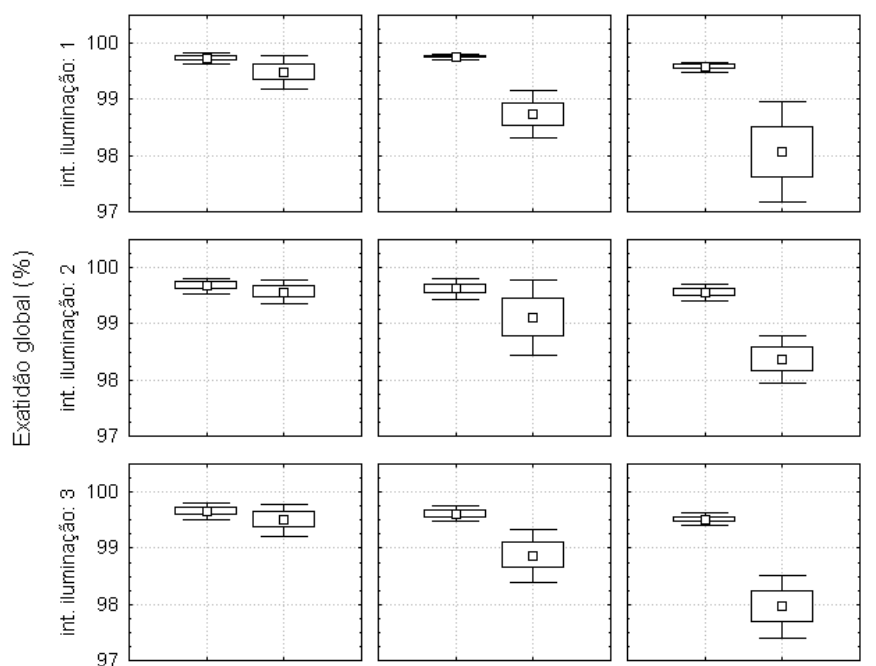

B.
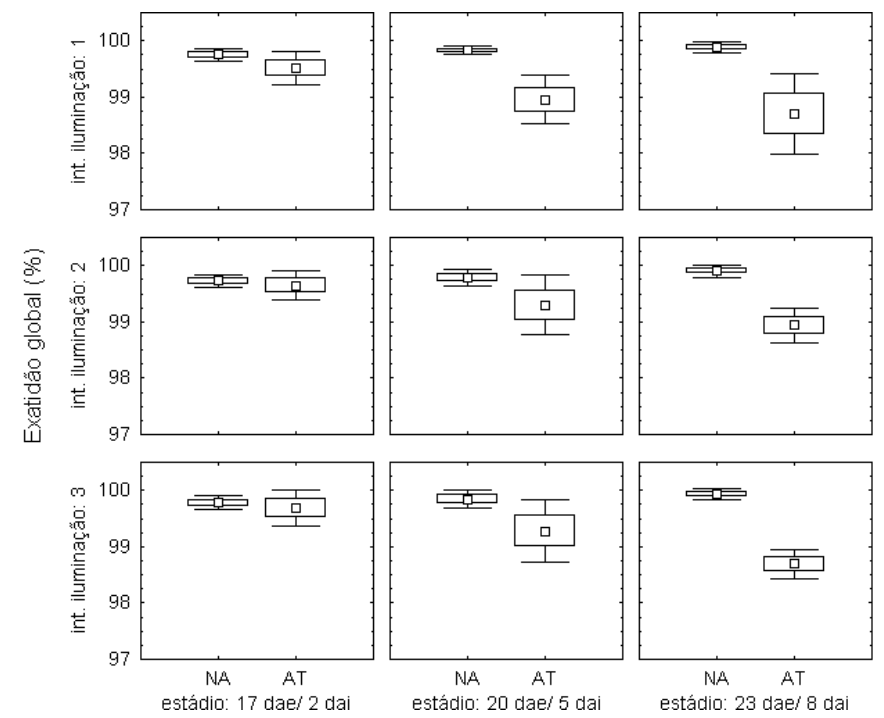

$\mp \pm 1.96^{\star}$ desv pad

$\pm 1.00^{*}$ desv. pad

- Média

Figura 2. Resultados da estatística descritiva dos valores da exatidão global das imagens binárias obtidas com o algoritmo bayesiano (A) e interativo (B), considerando-se o limiar manual como referência, para plantas não atacadas (NA) e atacadas (AT) em 3 épocas de obtenção das imagens e sob 3 intensidades de iluminação

plantas atacadas, a exatidão foi superior nos estádio 17 dae/ 2 dai com ligeira redução da exatidão, à medida que as plantas se desenvolveram e as injúrias se tornaram maiores. Praticamente, não se notou efeito da intensidade de iluminação, evidenciando a eficácia do índice do excesso de verde normalizado. As influências mais marcantes foram o estádio de desenvolvimento e a presença das injúrias provocadas pela lagarta do cartucho.

Os resultados foram considerados satisfatórios, uma vez que se encontram trabalhos, como o de Ling \& Ruzhitsky (1996) em que um método automático de limiarização foi considerado eficiente, com diferenças de -2 a 6\%, comparando-se as áreas dos objetos determinados manualmente e na limiarização automática.
A Figura 3 ilustra a normalização e limiarização da imagem de uma planta de milho atacada pela lagarta do cartucho.

A.

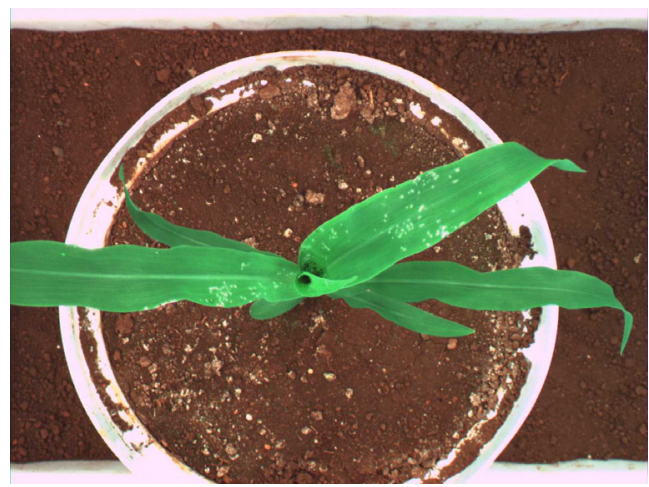

B.

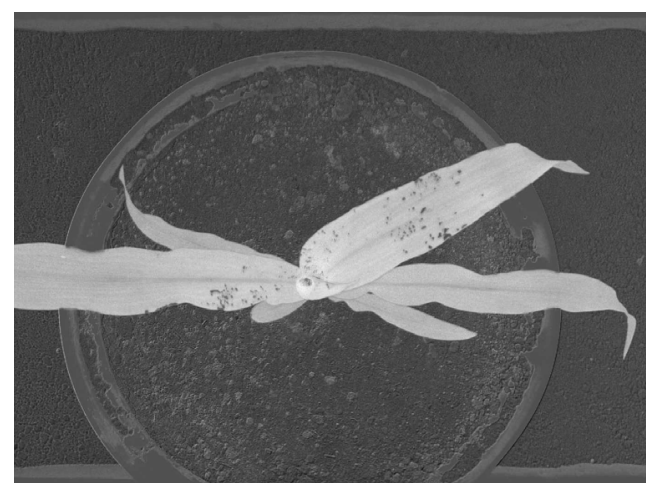

C.

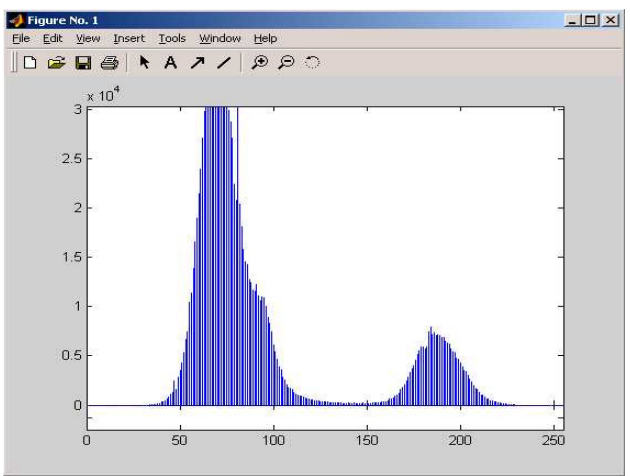

D.

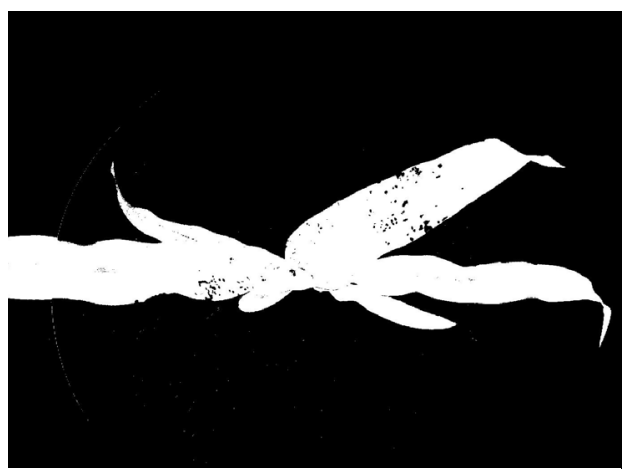

Figura 3. Imagem original para uma planta atacada pela lagarta do cartucho (A); imagem processada com o índice do excesso de verde normalizado (B); histograma da imagem processada e o limiar manual utilizado para segmentação (C); imagem binária resultante da segmentação com o limiar manual (D) 
Na imagem processada (Figura 3B), os pixels representando folhas apresentam-se em tons mais claros que o restante da imagem, inclusive as bordas do vaso, que aparecem em branco na imagem original (Figura 3A). Este resultado é importante pois, em condições de campo, especialmente em plantas cultivadas em sistema de plantio direto, a palha sobre o solo vai aparecer em tom mais claro que as folhas nas imagens originais, da mesma forma que as bordas do vaso nesta imagem.

No histograma da imagem processada (Figura 3C), nota-se a clara distinção das duas classes, separadas por um vale acentuado, mostrando a eficácia do índice do excesso de verde normalizado para realçar os pixels de folhas. Este realce ocorreu devido às características espectrais das folhas na faixa do visível, em que os pigmentos presentes, sobretudo a clorofila, absorvem intensamente a energia nas bandas vermelha e azul, enquanto refletem na banda verde. Este fato não ocorre nos demais componentes da imagem (solo, porções atacadas das folhas e bordas do vaso) onde a relação entre as bandas e, conseqüentemente, entre os valores dos pixels, é mais equilibrada.

A Figura 4 ilustra a segmentação da mesma imagem da planta da Figura 3, utilizando-se o limiar iterativo e o limiar bayesiano.

A.

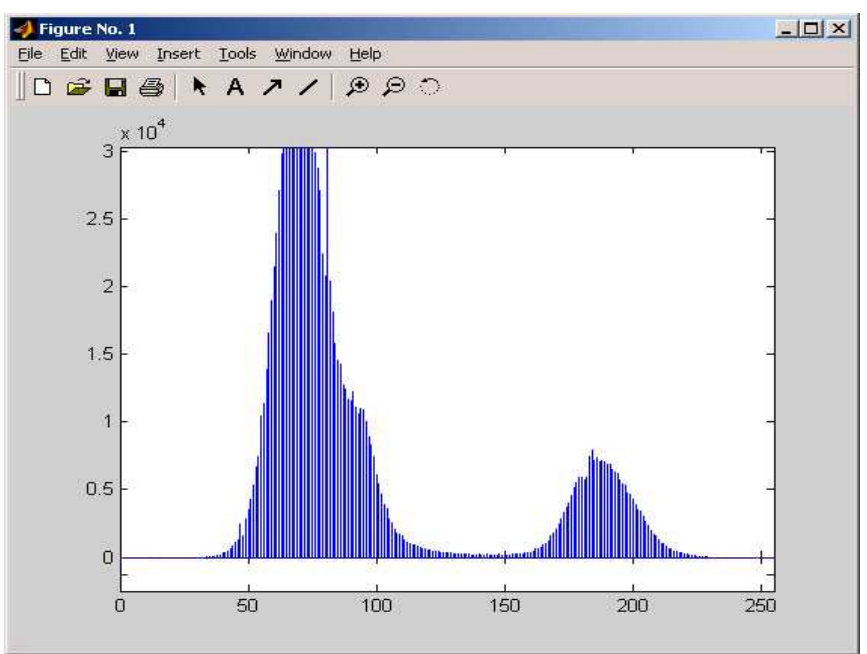

C.

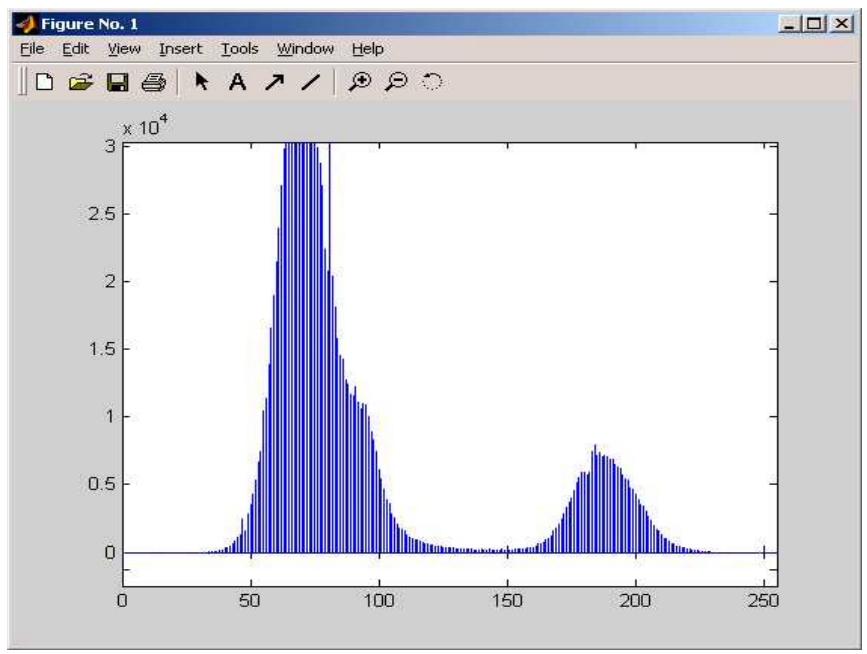

Yang et al. (2001) utilizaram a proximidade do valor do limiar, definido automaticamente com o valor do limiar manual como parâmetro para avaliar os métodos automáticos de limiarização. No presente trabalho, embora os valores dos limiares definidos pelos métodos automáticos tenham sido diferentes, em termos numéricos, dos limiares manuais, as imagens binárias foram muito semelhantes, conforme demonstrado pela exatidão global, devido à boa separação entre as classes. Ao se alterar o valor do limiar dentro do vale, o número de pixels atribuído à outra classe é pequeno em relação ao número total de pixels da imagem.

A Figura 5 ilustra as imagens-diferença de uma planta atacada e de uma planta não-atacada pela lagarta do cartucho, segmentadas com o método iterativo, em relação à limiarização manual. Nessas imagens são apresentados, em preto, os pixels classificados de forma diferente pelo método iterativo em relação ao método manual. Verifica-se que, em geral, o erro da limiarização automática ocorreu nas bordas - das folhas, das injúrias provocadas pela lagarta e do vaso - na transição de um objeto para outro. Quando se altera o valor do limiar, os pixels que se localizam no vale do histograma são atribuídos a outra classe. Normalmente, esses pixels são os das bordas, pois a transição

B.

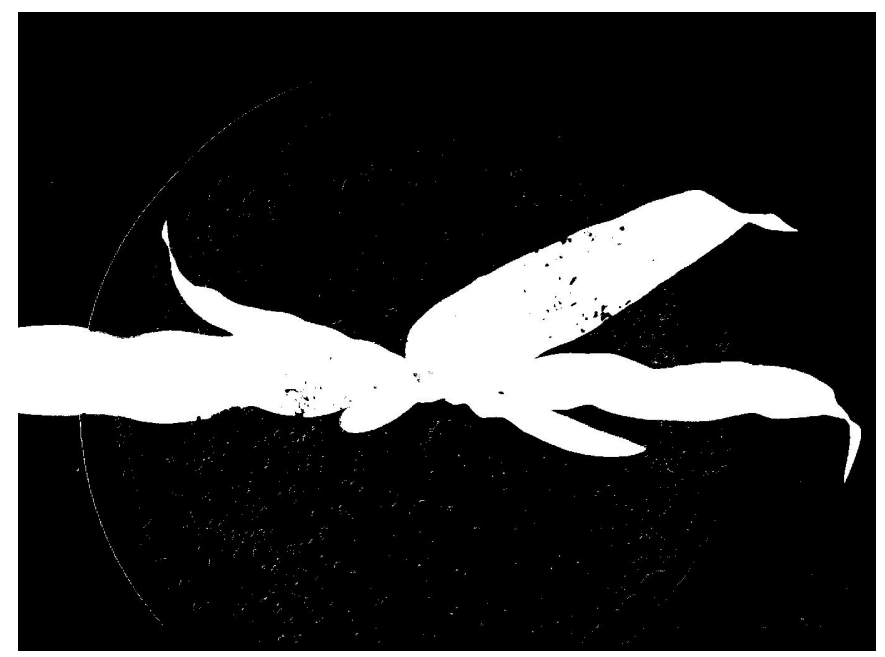

D.

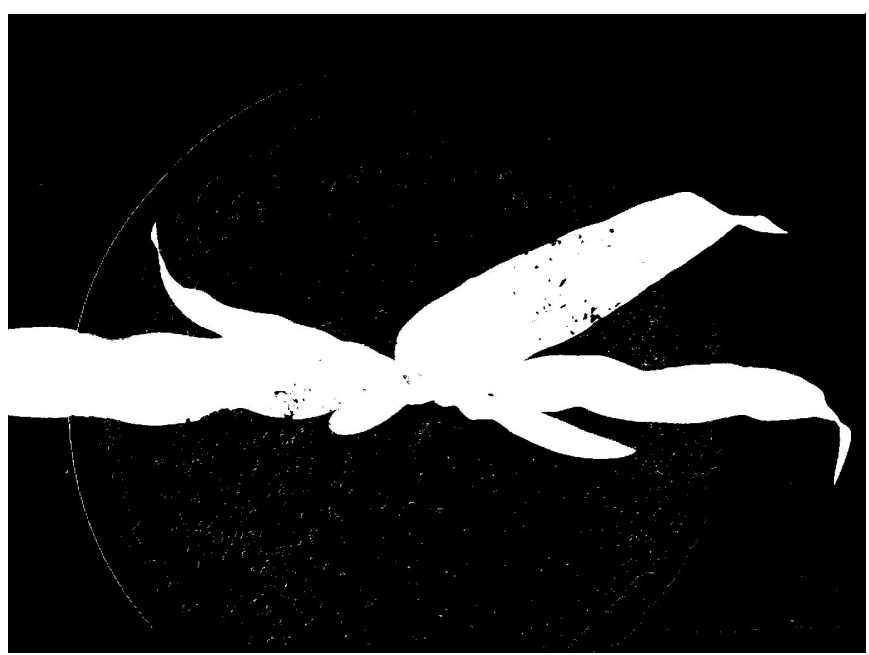

Figura 4. Histograma da imagem processada indicando o valor do limiar iterativo (A); imagem binária criada com o limiar iterativo (B); histograma da imagem processada indicando o valor do limiar bayesiano (C); imagem binária criada com o limiar bayesiano (D) 
A.

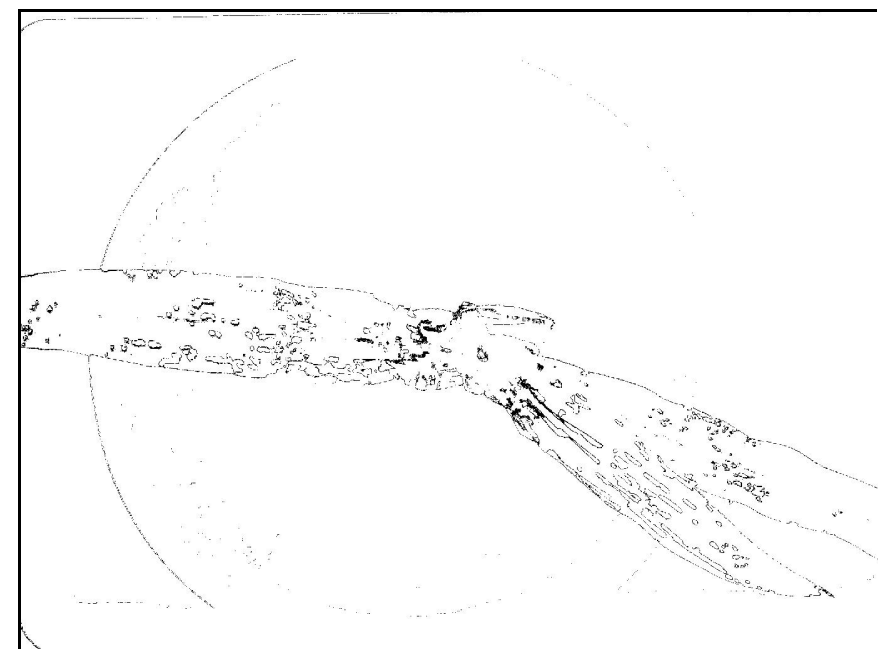

B.

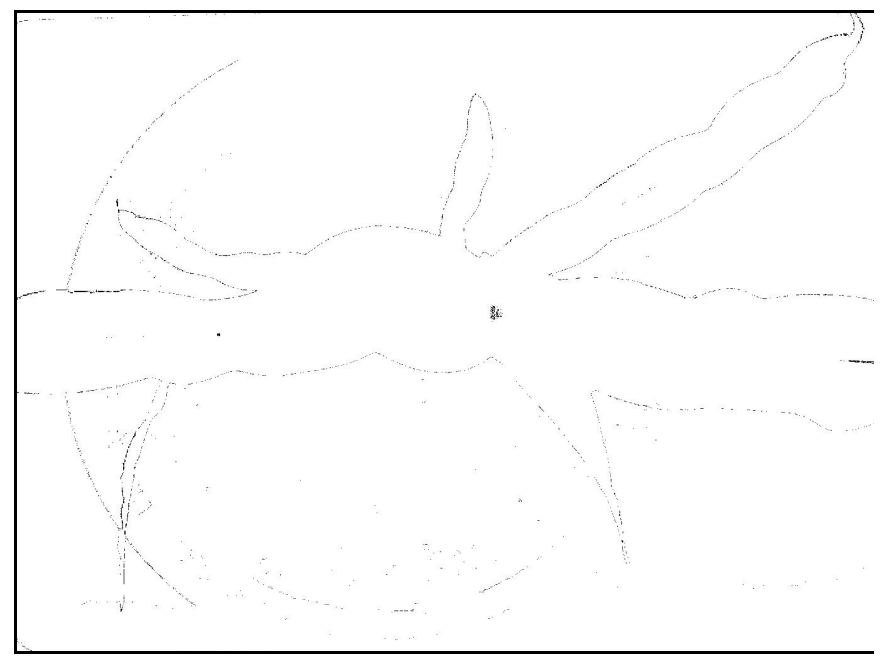

Figura 5. Imagem-diferença de uma planta atacada segmentada com o limiar iterativo em relação à limiarização manual (A) e imagemdiferença de uma planta não atacada segmentada com o limiar iterativo em relação à limiarização manual (B)

entre uma classe e outra é gradual, em termos de níveis de cinza. As imagens de plantas atacadas nos estádios mais avançados apresentaram exatidão global ligeiramente menor na classificação devido, sem dúvida, ao maior número de objetos e, conseqüentemente, maior número de pixels de transição (bordas).

Com esses resultados, acredita-se que é possível utilizar-se tanto o algoritmo iterativo quanto o bayesiano para segmentar, automaticamente, imagens de plantas de milho processadas com o índice do excesso de verde normalizado. A principal vantagem da utilização do algoritmo iterativo é sua menor exigência, em termos computacionais, em relação ao algoritmo bayesiano, o que facilita sua implementação em sistemas de visão artificial.

\section{CONCLUSÕES}

1. A intensidade de iluminação foi o fator estudado que apresentou menor influência sobre a exatidão global da classificação, comparando-se as imagens binárias dos métodos de limiarização automática com a limiarização manual.

2. Os fatores-ataque (plantas atacadas ou não-atacadas) e época (dias após a emergência/infestação) apresentaram maior influência sobre a exatidão global da classificação, devido ao maior número de pixels de transição entre os objetos (bordas) que foram classificados incorretamente.

3. Os dois algoritmos de limiarização automática (iterativo e bayesiano) têm potencial para serem utilizados em um sistema de visão artificial, para identificação de plantas de milho atacadas pela lagarta do cartucho. $\mathrm{O}$ algoritmo bayesiano, no entanto, é mais exigente, em termos computacionais, que o algoritmo iterativo.

\section{AGRADECIMENTOS}

Aos pesquisadores, técnicos e demais funcionários da EMBRAPA Milho e Sorgo, em Sete Lagoas, MG, por viabilizarem o desenvolvimento desta pesquisa. À Fundação de Amparo à Pesquisa do Estado de Minas Gerais (FAPEMIG) pelo financiamento concedido ao primeiro autor, durante o curso de
Mestrado. Pesquisa financiada pelo Banco Mundial e EMBRAPA, através do Projeto de Apoio ao Desenvolvimento de Tecnologia Agropecuária para o Brasil (PRODETAB Projeto 030-01/99)

\section{LITERATURA CITADA}

Andreadis, I.; Tsalides, P. Analog computation of image chromaticity. Real-Time Imaging, London, n.3, p.1-6, 1997.

Batlle, J.; Casals, A.; Freixenet, J.; Martí, J. A review on strategies for recognizing natural objects in colour images of outdoor scenes. Image and Vision Computing, Amsterdam, n. 18, p.515-530, 2000.

Carvalho, R.P.L. Pragas do milho.In: Paterniani, E.; Viégas, G.P. (ed.) Melhoramento e produção de milho. v.2. 2 ed. Campinas: Fundação Cargill, 1987. p.637-712 .

Fancelli, A.L.; Dourado Neto, D. Produção de milho. 1 ed. Guaíba: Livraria e Editora Agropecuária, 2000, 360p.

Gonzales, R.C.; Woods, R.E. Digital image processing. Reading, Massachusetts: Addison-Wesley Publishing Company, 1992, 716p.

Jensen, J.R. Introductory digital image processing: A remote sensing perspective. New York: Prentice Hall, 1996, 318p.

Ling, P.P.; Ruzhitsky, V.N. Machine vision techniques for measuring the canopy of tomato seedling. Journal of Agricultural Engineering Research, London, v.65, p.85-95, 1996.

Marques Filho, O.; Vieira Neto, H. Processamento digital de imagens. Rio de Janeiro: Brasport, 1999, 406p.

Mateus, G.R.; Luna, H.P. Programação não linear. Belo Horizonte: Editora Gráfica Formato Ltda, 1986, 289p.

Meyer, G.E.; Mehta, T.; Kocher, M.F.; Mortensen, D.A.; Samal, A. Textural imaging and discriminant analysis for distinguishing weeds for spot spraying. Transactions of the ASAE, St. Joseph, v.41 n.4, p.1189-1197, 1998.

Oliveira, J.P.; Silva, A.L. Controle químico da lagarta do cartucho (Spodoptera frugiperda) em milho, com novo regulador de crescimento. In: Congresso Nacional de Milho e Sorgo, $2^{\circ}$, 1994, Goiânia, Resumos... Goiânia: Associação Brasileira de Milho e Sorgo, 1994. 162p. 
Oliveira, L.J. Biologia, nutrição quantitativa e danos causados por Spodoptera frugiperda (J.E. Smith, 1797) (lepidóptera: noctuidae) em milho cultivado em solo corrigido para três níveis de alumínio. Piracicaba: ESALQ, 1987. 125p. Dissertação Mestrado

Pinto, F.A.C.; Sena Jr, D.G.; Queiroz, D.M.; Gomide, R.L. Visão artificial na agricultura de precisão. In: Balastreire (ed.) Avanços na Agricultura de Precisão no Brasil no período 1999-2001, Piracicaba: L.A. Balastreire, 2002, p.309-316.

Stewart, B.L.; Tian, L.F. Real-time machine vision weed-sensing. ASAE paper 98 - 3033, ASAE, 1998. CD-Rom

Tian, L.F.; Slaughter D.C. Environmentally adaptive segmentation algorithm for outdoor image segmentation. Computers and Electronics in Agriculture, Oxford, n.21, p.153-168, 1998.
Viana, P.A.; Costa, E.F. Eficiência de inseticidas misturados em óleo vegetal aplicados via irrigação por aspersão para o controle da lagarta do cartucho Spodoptera Frugiperda em milho. In: Congresso Nacional de Milho e Sorgo, 2 1994 Goiânia, Resumos... Goiânia: Associação Brasileira de Milho e Sorgo, 1994. p.138.

Woebbecke, D.M.; Meyer, G.E.; von Bargen, K.; Mortensen, D.A. Color indices for weed identification under various soil, residue, and lighting conditions. Transactions of the ASAE. St. Joseph, v.38, n.1, p.259-269, 1995.

Yang, L.; Albregtsen, F.; Lonnestad, T.; Grottum, P. A supervised approach to the evaluation of image segmentation methods. Computer Analysis of Images and Patterns, Prague, 1995, p.759-765.

Yang, X; Beyenal, H.; Gary H.; Lewandowski, Z. Evaluation of biofilm image thresholding methods. Water Research, Colchester, v.35, p.1149-1158, 2001. 